\title{
JRRD -
}

\section{Elevated liver enzymes following polytraumatic injury}

\author{
Aaron Fox, MD; ${ }^{*}$ James B. Sanderlin, MD; $^{2}$ Shane McNamee, MD; ${ }^{2}$ Jasmohan S. Bajaj, MD; $^{3}$ William Carne, \\ PhD; ${ }^{2}$ David X. Cifu, MD ${ }^{2}$ \\ Departments of ${ }^{1}$ Primary Care, ${ }^{2}$ Physical Medicine and Rehabilitation, and ${ }^{3}$ Gastroenterology/Hepatology, Hunter \\ Holmes McGuire Department of Veterans Affairs Medical Center, Richmond, VA
}

\begin{abstract}
This retrospective cohort study examined the prevalence and potential risk factors for elevated liver enzymes in patients following traumatic brain injury (TBI). The participants were servicemembers with TBI admitted to the Polytrauma Rehabilitation Center (PRC) at the Hunter Holmes McGuire Department of Veterans Affairs Medical Center in Richmond, Virginia, from January 2008 through December 2011. The PRC had 207 patients during this time period, 121 of whom had a liver panel within $30 \mathrm{~d}$ of injury. Patients were retrospectively analyzed and placed into one of two categories based on alanine aminotransferase (ALT) values. Of the 121 subjects, 59 (49\%) had an ALT of 44 IU/L or greater on their initial set of laboratories. These subjects were compared with those with an ALT of $43 \mathrm{IU} / \mathrm{L}$ or less using chi square analysis. There were no significant differences between the two groups with regard to sex, military status, race, theater, TBI mechanism, severity of TBI, or concomitant injuries. Regardless of demographics, mechanism of injury, or extent of trauma, elevated liver enzymes were common in patients admitted to the rehabilitation unit following TBI. For the majority of these patients, enzymes returned to normal with conservative management. In most cases, no specific etiology was ever defined. Further analysis will be performed to determine the most efficient way to monitor these patients so that unnecessary tests are avoided and medical expenses are minimized.
\end{abstract}

Key words: blast injuries, brain injury, drug-induced liver injuries, liver enzyme elevations, liver function tests, polytraumatic injury, rehabilitation, TBI, trauma severity indices, Veteran.

\section{INTRODUCTION}

The signature injury of the recent Middle East conflicts has been identified as traumatic brain injury (TBI); however, this may be better characterized as polytrauma injury [1]. Because of the violent nature of these events, patients with TBI almost always have a complex pattern of concomitant injuries extending beyond the brain. To deliver the best possible care for Veterans and injured Active Duty servicemembers with polytraumatic injuries,

\footnotetext{
Abbreviations: ALT $=$ alanine aminotransferase, $\mathrm{DOD}=$ Department of Defense, PRC $=$ Polytrauma Rehabilitation Center, TBI = traumatic brain injury, ULN = upper limit of normal, VA $=$ Department of Veterans Affairs, VAMC = VA Medical Center.

*Address all correspondence to Aaron Fox, MD; Hunter Holmes McGuire VAMC-Primary Care Service, 1201 Broad Rock Blvd, Richmond, VA 23249; 804-675-5000; fax: 804-675-5028. Email: aaron.fox@va.gov
}

http://dx.doi.org/10.1682/JRRD.2013.10.0233 
it is important to develop a rational approach to common medical complications following TBI.

One of the more prevalent laboratory abnormalities following TBI is mildly elevated liver enzymes [2]. While liver enzyme testing is often useful for monitoring individuals with known liver diseases, the clinical significance and specificity of abnormal values are not always clear in the general population [3]. Finding elevated liver enzymes usually prompts a cascade of diagnostic testing to include repeat serum analysis, imaging, and potentially invasive testing such as biopsy [4]. Often these tests are unrevealing, leaving the clinician to decide between additional testing versus continued monitoring.

The purpose of this single-site study was to describe and examine factors associated with mild liver enzyme elevations in patients with TBI. We reviewed the pattern, natural history, and results of the workup performed in a cohort of patients with TBI. This information may assist clinicians in the evaluation and management of individuals with TBI found to have elevated liver enzymes.

\section{METHODS}

\section{Sample}

Inclusion criteria for the sample were all servicemembers with TBI admitted to the Hunter Holmes McGuire Department of Veterans Affairs (VA) Medical Center (VAMC) Polytrauma Rehabilitation Center (PRC) from January 2008 through December 2011. The PRC had 207 patients during this time period and 121 of these patients had a liver panel within $30 \mathrm{~d}$ of injury.

\section{Measures}

A physician chart reviewer manually extracted data from the VA and Department of Defense (DOD) Electronic Medical Records and inputted data directly into an Excel (2010, Microsoft Corporation; Redmond, Washington) spreadsheet. Results from up to 10 liver panels following injury were recorded. Severity of TBI was determined using the DOD/VA Traumatic Brain Injury Task Force recommendations [5]. Other recorded data included demographics, mechanism of TBI, timing of injury, concomitant injuries, and workup performed as the result of elevated liver enzymes. Patients were retrospectively analyzed and placed into one of two categories based on alanine aminotransferase (ALT) values. Because a generally accepted upper limit of normal
(ULN) does not exist for ALT, we used a threshold of $44 \mathrm{IU} / \mathrm{L}$ as abnormal in men, as reported in the most recent National Health and Nutrition Examination Survey data [6].

\section{Statistical Analysis}

Data were analyzed using SPSS 16.0 (IBM Corporation; Armonk, New York). Descriptive statistics were calculated to characterize the sample. Chi square analysis was used to examine observed categorical data relative to expected frequencies. A $p$-value of $<0.05$ was set as being statistically significant.

\section{RESULTS}

General characteristics of the patients are summarized in Table 1. Inclusion criteria were met by 121 subjects. The majority of these patients were Active Duty Caucasian males, with 54 percent sustaining their TBI in the Middle East. Sixty-two percent had a severe TBI (43\% classified as severe and $19 \%$ as penetrating). Thirty-six percent of the TBIs were the result of a blast.

Of the 121 subjects, 59 (49\%) had an ALT >44 IU/L on their initial set of laboratories. These subjects were grouped and compared with those with an ALT $<43$ IU/L ( $n=56)$ using chi square analysis. There were no significant

Table 1.

Baseline characteristics of study sample.

\begin{tabular}{lc}
\hline \multicolumn{1}{c}{ Characteristic } & Value \\
\hline Days from Injury to Admission (mean) & 32.9 \\
Male/Female $(n)$ & $115 / 6$ \\
Military: Active Duty/Veteran $(n)$ & $106 / 15$ \\
Race $(n)$ & \\
Caucasian & 102 \\
African-American & 11 \\
Hispanic & 4 \\
Other & 4 \\
Locale $(n)$ & \\
Iraq & 15 \\
Afghanistan & 50 \\
United States & 51 \\
Other & 5 \\
Traumatic Brain Injury Severity $(n)$ & \\
Mild & 26 \\
Moderate & 33 \\
Severe & 43 \\
Penetrating & 19
\end{tabular}


Table 1.

Baseline characteristics of study sample.

Traumatic Brain Injury Mechanism (n)

Closed 58

Penetrating (Gunshot/Stabbing) 14

Blast

Other (Anoxia)

$\geq 2$ Causes with Blast

$\geq 2$ Causes Without Blast

Liver Trauma: Performed/Abnormal (n)

$17 / 4$

differences between the two ALT groups with regards to sex, military status, race, theater, TBI mechanism, severity of TBI, or concomitant injuries (Table 2).

The average ALT in the case group was 89 IU/L. In this group, 28 of 59 subjects (47\%) had an ALT $\leq 2$ times the ULN, 26 of the 59 subjects (44\%) had an elevation 2 5 times the ULN, and 5 subjects (9\%) had an elevation 5 10 times the ULN. No subject had a value $\geq 10$ times the ULN. The highest initial ALT was 403 IU/L. Four subjects had a ratio of aspartate aminotransferase/ALT $\geq 2$. One subject had a bilirubin level $\geq 2 \mathrm{mg} / \mathrm{dL}$.

By the time of discharge, 41 of the 59 (69\%) patients had improvement or resolution of the liver enzymes. Repeat testing was not performed for 11 of the 59 (19\%). ALT worsened when compared with their initial value for 7 of the 59 (12\%). One patient was found to have a sustained elevation $>5$ times the ULN on discharge.

The workup performed is summarized in Table 3. Eighteen subjects (30\%) had hepatitis C testing, but only one patient was confirmed to have antibodies to hepatitis
C. Thirteen subjects had testing for hepatitis B and none showed active disease; however, one patient had a prior exposure. Eight subjects had negative hepatitis A testing. Two subjects had negative antinuclear antibody testing. Fifteen subjects had creatinine kinase enzymes assessed and although six subjects had values $\geq 1,000 \mathrm{IU} / \mathrm{L}$, this finding was not addressed in relation to the elevated ALT. Six subjects had screening laboratories for hemochromatosis, and one had a positive screen (Fe [iron] saturation $>45 \%$ and ferritin $>200 \mathrm{ng} / \mathrm{mL}$ ); however, it was not mentioned in relation to the elevated ALT. Nine subjects (15\%) had liver ultrasound, and two were considered abnormal.

Of the 59 subjects, 5 (8\%) had a formal diagnosis in the chart for the liver enzyme elevation. Four were attributed to medications and one to direct liver trauma. There was no documentation in the chart regarding the diagnosis of hepatitis $C$ in the servicemember who screened positive. Twelve subjects (20\%) had mention of the ALT elevation in the chart, but no formal diagnosis was made. No mention was made of the liver enzyme elevation for 42 of the 59 subjects (71\%). Finally, 33 of the 59 patients (56\%) had $\geq 5$ liver enzymes assessments and 12 (20\%) had $\geq 10$.

\section{DISCUSSION}

This single-center pilot study not only reflects that mild elevations in liver enzymes are common in the population

Table 2.

Correlation between serum alanine aminotransferase (ALT) level and qualitative factors.

\begin{tabular}{|c|c|c|c|}
\hline Factor & $<43$ ALT & $>44$ ALT & p-Value \\
\hline Caucasian/African American/Hispanic/Other $(n)$ & $50 / 6 / 3 / 3$ & $52 / 5 / 1 / 1$ & 0.56 \\
\hline Closed/Penetrating/Blast/Other/ $\geq 2$ with Blast/ $\geq 2$ Without Blast $(n)$ & 28/9/11/1/12/1 & $30 / 5 / 9 / 4 / 10 / 1$ & 0.65 \\
\hline Long Bone Fracture: 0/1/2 $(n)$ & $41 / 10 / 11$ & $36 / 8 / 14$ & 0.68 \\
\hline Liver Trauma: No/Yes $(n)$ & $61 / 1$ & $56 / 3$ & 0.29 \\
\hline
\end{tabular}

Table 3.

Workup for elevated liver enzymes. 
JRRD, Volume 51, Number 6, 2014

Table 3.

Workup for elevated liver enzymes.

Hepatitis A Immunoglobulin M Antibody

Hepatitis B Core Antibody

Hepatitis B Surface Antigen

$8 / 0$

Hepatitis C Antibody

Antinuclear Antibody

Fe Saturation $>45 \%$ and Ferritin $>200$ ng/mL

Creatinine Phosphokinase

Liver Imaging

13/1 (no mention of this abnormality)

$15 / 0$

$18 / 1$ (no mention of this abnormality)

$2 / 0$

6/1 (no mention of this abnormality)

15/6 (no mention of this abnormality)

9/2 (gall bladder contraction, right hepatic lobe injury)

of servicemembers with TBI, but also illustrates a need for consistency in the identification and workup of this finding.

In our cohort of patients, the pattern of liver enzyme elevation was uniformly hepatocellular. Only one patient had a bilirubin level greater than $2 \mathrm{mg} / \mathrm{dL}$. Commonly worked-up causes of this pattern in the general population include viral hepatitis, hemochromatosis, autoimmune hepatitis, and Wilson disease. The most common of these is hepatitis $\mathrm{C}$, with an estimated prevalence of 1.6 percent in the general population [7].

Although an algorithmic approach relying on a host of laboratories may seem appealing, we advocate against this in favor of an approach that considers the clinical context of the patient. Initiating a thorough workup in every patient with a mild elevation in ALT would be time consuming, costly, and likely unrevealing. In addition, this strategy could potentially signal an additional "problem" in roughly half the patients admitted to the inpatient rehabilitation service. This may erroneously lead to additional and unnecessary anxiety for the patient and family, who are trying to focus on rehabilitation following a debilitating injury.

In the absence of risk factors, signs of decompensated liver failure on examination, or chronically elevated liver enzymes, we do not recommend routine screening for one of these uncommon causes. In addition, we do not recommend routine use of liver ultrasound. In our study, nine patients underwent liver ultrasound. Although two were considered abnormal, none led to a specific diagnosis. Sanfilippo et al. retrospectively looked at the usefulness of liver ultrasound in the evaluation of isolated liver function test abnormalities in patient with TBI in the intensive care unit setting [8]. Even with a mean ALT (246 IU/L) significantly higher than our threshold for abnormal, it was not helpful in identifying the cause.

Sanfilippo et al. implicated TBI, critical illness, and pharmacology as likely causes in these patients [8]. Each of these explanations are plausible in our population, and a natural extension of this pilot study will be looking at additional liver panels to see trends in ALT as the Veteran recovers from the injury. If the initial liver panel demonstrates an elevated ALT in the acute period following the TBI, this would favor a spectrum of liver injury associated with critical illness, as suggested by Thompson et al. [9]. However, if liver enzyme elevations develop or worsen in the days and weeks that follow, a more likely explanation in this patient population is drug-induced liver injury. Patients with TBI are often on multiple medications, including antibiotics, antidepressants, acetaminophen, and anticonvulsants, which can cause a subclinical elevation in ALT that tends to resolve once the medication has been discontinued [10]. Although a formal diagnosis was rarely made in this study, four of the five diagnoses that were documented implicated medications.

This single-center pilot study has several shortcomings that limit us from generalizing these results to all patients with TBI. The sample is too small and too sex biased to allow for meaningful inferential conclusions regarding the incidence of liver problems in TBI patients nationally. Our medical center is a teaching hospital, and over the $4 \mathrm{yr}$ analyzed, we had multiple residents and fellows who rotated through the inpatient rehabilitation service. This may in part explain some of the variability with regard to workup and identification of laboratory abnormalities. Next, the majority of patients studied were transferred from DOD facilities. Although effective transfer of information between DOD and the VA has greatly improved recently, some information, including level of care at the time of initial liver function testing, may not have been available for review by the authors. Finally, it should be noted that no consensus exists regarding the normal range for ALT and reference ranges differ in various settings [11]. We used a value of $44 \mathrm{IU} / \mathrm{L}$, which has 95 percent specificity for predicting those at low risk for liver injury; however, the ULN for ALT at our medical center is $78 \mathrm{IU} / \mathrm{L}$. This value is considerably 
higher because it is based on the central 95 percent of the general population of Veterans at our institution, many of whom are older with liver disease. If this value were used, 23 of the 59 (39\%) subjects considered to have elevated ALT would be reclassified as having a normal value.

While these limitations restrict the applicability of these data to other settings and populations, this pilot study demonstrates that liver testing needs to be carefully considered within the clinical context of the individual patient.

\section{CONCLUSIONS}

Regardless of demographics, mechanism of injury, or extent of trauma, mild elevations in ALT are a common and often undiagnosed finding following TBI. Our study demonstrated that roughly one-half of the patients with TBI admitted to the Hunter Holmes McGuire VAMC PRC from 2008 to 2011 had an ALT $\geq 44$ IU/L within the first $30 \mathrm{~d}$ following injury. The liver enzyme elevations were mild to moderate ( $\leq 5$ times ULN) and rarely was a formal diagnosis established. Most enzyme elevations either resolved, or were resolving, by the time of discharge without specific intervention and were presumed to be due to medications in the absence of other etiologies. None of the patients developed acute liver injury or failure as a result of these presumed medication-associated liver injuries. Further analysis will be performed to analyze the most efficient way to monitor these patients so that unnecessary tests are avoided and medical expenses are kept to a minimum.

\section{ACKNOWLEDGMENTS}

\section{Author Contributions:}

Study concept and design: A. Fox, S. McNamee, W. Carne, D. X. Cifu. Assisted with literature review: J. S. Bajaj.

Assisted with data collection: J. B. Sanderlin.

Prepared "Discussion" section of manuscript: J. S. Bajaj.

Directed study, including quality assurance and control: A. Fox.

Financial Disclosures: The authors have declared that no competing interests exist.

Funding/Support: This material was unfunded at the time of manuscript preparation.

Institutional Review: The authors obtained institutional review board approval for this study from the Hunter Holmes McGuire VAMC.

Participant Follow-Up: The authors do not plan to inform participants of the publication of this study.

\section{REFERENCES}

1. Cifu DX, Taylor BC, Carne WF, Bidelspach D, Sayer NA, Scholten J, Campbell EH. Traumatic brain injury, posttraumatic stress disorder, and pain diagnoses in OIF/OEF/OND Veterans. J Rehabil Res Dev. 2013;50(9):1169-76. [PMID:24458958] http://dx.doi.org/10.1682/JRRD.2013.01.0006

2. Kalisky Z, Morrison DP, Meyers CA, Von Laufen A. Medical problems encountered during rehabilitation of patients with head injury. Arch Phys Med Rehabil. 1985;66(1):25-29. [PMID:3917661]

3. Goessling W, Friedman LS. Increased liver chemistry in an asymptomatic patient. Clin Gastroenterol Hepatol. 2005; 3(9):852-58. [PMID:16234021] http://dx.doi.org/10.1016/S1542-3565(05)00416-7

4. Ioannou GN, Boyko EJ, Lee SP. The prevalence and predictors of elevated serum aminotransferase activity in the United States in 1999-2002. Am J Gastroenterol. 2006; 101(1):76-82. [PMID:16405537] http://dx.doi.org/10.1111/j.1572-0241.2005.00341.x

5. Department of Defense and Department of Veterans Affairs. Traumatic Brain Injury Task Force [Internet]. Atlanta (GA): Centers for Disease Control and Prevention; 2008. Available from: http://www.cdc.gov/nchs/data/icd/ Sep08TBI.pdf

6. Ruhl CE, Everhart JE. Upper limits of normal for alanine aminotransferase activity in the United States population. Hepatology. 2012;55(2):447-54. [PMID:21987480] http://dx.doi.org/10.1002/hep.24725

7. Armstrong GL, Wasley A, Simard EP, McQuillan GM, Kuhnert WL, Alter MJ. The prevalence of hepatitis $C$ virus infection in the United States, 1999 through 2002. Ann Intern Med. 2006;144(10):705-14. [PMID:16702586] http://dx.doi.org/10.7326/0003-4819-144-10-200605160-00004

8. Sanfilippo F, Veenith T, Santonocito C, Vrettou CS, Matta BF. Liver function test abnormalities after traumatic brain injury: Is hepato-biliary ultrasound a sensitive diagnostic tool? Br J Anaesth. 2014;112(2):298-303.

[PMID:24067331] http://dx.doi.org/10.1093/bja/aet305

9. Thomson SJ, Cowan ML, Johnston I, Musa S, Grounds M, Rahman TM. 'Liver function tests' on the intensive care unit: A prospective, observational study. Intensive Care Med. 2009;35(8):1406-11. [PMID:19513695] http://dx.doi.org/10.1007/s00134-009-1511-7

10. Navarro VJ, Senior JR. Drug-related hepatotoxicity. N Engl J Med. 2006;354(7):731-39. [PMID:16481640] http://dx.doi.org/10.1056/NEJMra052270

11. Dutta A, Saha C, Johnson CS, Chalasani N. Variability in the upper limit of normal for serum alanine aminotransferase levels: A statewide study. Hepatology. 2009;50(6): 
JRRD, Volume 51, Number 6, 2014

1957-62. [PMID:19787805]

http://dx.doi.org/10.1002/hep.23200

Submitted for publication October 28, 2013. Accepted in revised form January 22, 2014.

This article and any supplementary material should be cited as follows:

Fox A, Sanderlin JB, McNamee S, Bajaj JS, Carne W, Cifu DX. Elevated liver enzymes following polytraumatic injury. J Rehabil Res Dev. 2014;51(6):869-74.

http://dx.doi.org/10.1682/JRRD.2013.10.0233

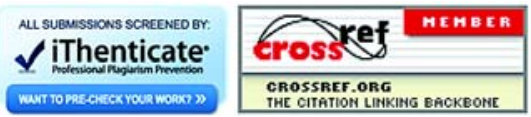


